\title{
Biosynthetic rivalry of o-aminophenol-carboxylic acids initiates production of hemi-actinomycins in Streptomyces antibioticus $\uparrow$
}

\begin{abstract}
Ivana Crnovčić, Siamak Semsary, Joachim Vater and Ullrich Keller*
Actinomycins consist of two pentapeptide lactone rings attached to 2-amino-4,6-dimethyl-3-oxophenoxazine-1,9-dicarboxylic acid (actinocin). The actinocin moiety is formed through oxidative condensation of two 3-hydroxy-4-methylanthranilic acid (4-MHA) pentapeptide lactones (actinomycin halves) as the last step of actinomycin biosynthesis. We found that feeding of 4-MHA or its putative biogenetic precursor 3-hydroxyanthranilic acid (3-HA) to Streptomyces antibioticus induced formation of different new compounds at the expense of actinomycins. These contain only one pentapeptide lactone ring attached to the $\beta$-side of their phenoxazinone ring systems and are formed through premature condensation of the externally added abundant 4-MHA or 3-HA with actinomycin halves. They were termed hemi-actinomycins and $\mathrm{C}$-demethyl-hemi-actinomycins, respectively, which differ from each other in the presence or absence of one or both methyl groups in their phenoxazinone moieties. 3-HA also induces synthesis of various $\mathrm{C}$-demethylactinomycins formed through condensation of actinomycin halves in which 3-HA had been incorporated by the 4-MHA incorporating enzyme in lieu of 4-MHA. 3-HA was not converted to 4-MHA as revealed by its inability to stimulate synthesis of actinomycin or hemi-actinomycin synthesis and thus remained a substrate analogue of 4-MHA rather than its precursor. In contrast to S. antibioticus, actinomycin-producing streptomycetes such as Streptomyces chrysomallus or Streptomyces parvulus do not form hemi-structured actinomycins when fed with 3-HA or 4-MHA. They do not possess the enzyme phenoxazinone synthase (PHS) which in S. antibioticus is present and most probably catalyses premature condensation of abundant 4-MHA or 3-HA with actinomycin halves. Testing hemi-acinomycin IV for drug activity revealed that it intercalates into DNA and inhibits relaxation and supertwisting of DNA by topoisomerase I and DNA-gyrase like actinomycin IV (D). Moreover, it has inhibitory activity on growth of Bacillus subtilis.
\end{abstract}

Received 8th October 2013 Accepted 6th December 2013

DOI: $10.1039 / \mathrm{c} 3 \mathrm{ra} 45661 \mathrm{~g}$

www.rsc.org/advances of nicotinic acid biosynthesis in all eukaryotes and a number of bacteria. ${ }^{4}$ However, in contrast to 3 -HA, 4-MHA formation is restricted to streptomycete strains which produce actinomycins and members of the diazepam group of natural compounds such as anthramycin, sibiromycin or tomaymycin all of which contain 4-MHA as building stone in their structures. ${ }^{5-7}$ The reaction sequences leading from tryptophan to 4-MHA or 3-HA differ only in the presence of an additional methylation step which introduces a methyl group into the benzene ring of 4-MHA (Fig. 1). In Streptomyces chrysomallus and Streptomyces parvulus which produce actinomycin $\mathrm{C}$ and actinomycin $\mathrm{D}$, respectively, 3-hydroxykynurenine (3-HK) is methylated by the 3-HK-methyltransferases AcmI/L to give 3-hydroxy-4-methylkynurenine (4-MHK) (Fig. 1, upper path). ${ }^{8}$-MHK is subsequently converted to 4-MHA by the hydroxykynureninase AcmH. ${ }^{8,9}$ The same steps of 4-MHA synthesis were also found in case of sibiromycin biosynthesis in Streptosporangium sibiricum. ${ }^{\mathbf{1 0}}$ By contrast, in actinomycin X-producing Streptomyces antibioticus, the methylation step appears to be catalyzed by a methyltransferase which methylates 3-HA directly giving 4-MHA
Technische Universität Berlin, Institut für Chemie, Müller-Breslau Strasse 10, D-10623 Berlin-Charlottenburg, Germany.E-mail: ullrich.keller@tu-berlin.de; Tel: +4930 314 25477

† Electronic supplementary information (ESI) available: Fig. S1-S7 and Tables S1, S2. See DOI: $10.1039 / \mathrm{c} 3 \mathrm{ra} 45661 \mathrm{~g}$ 


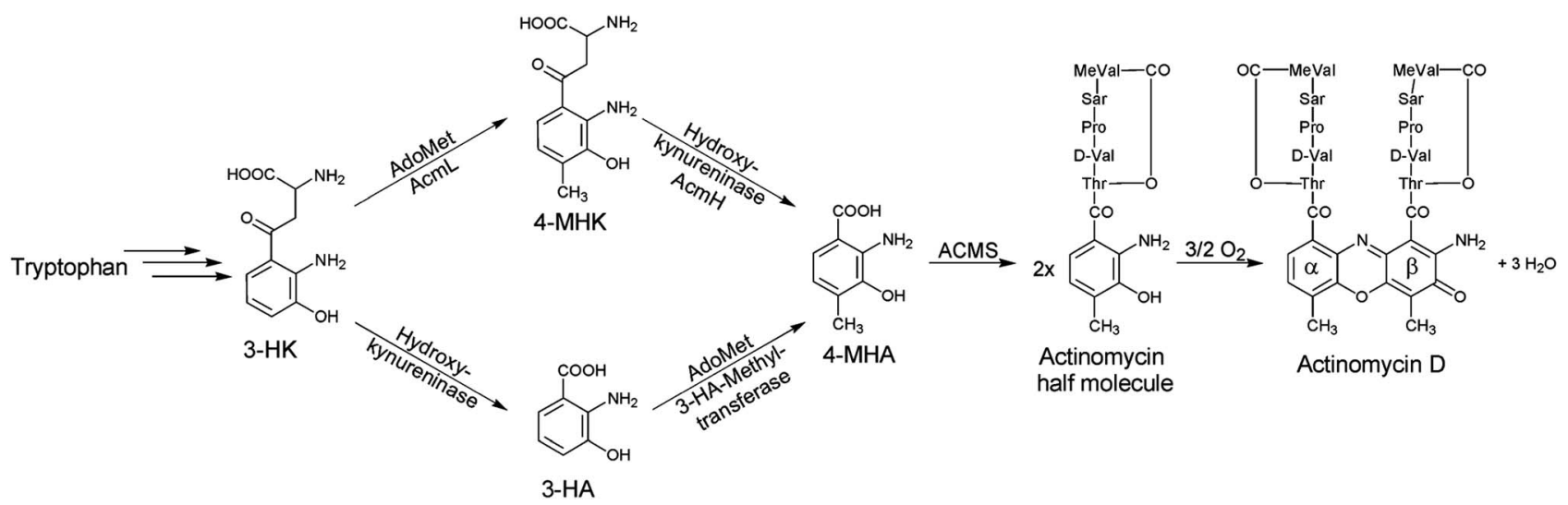

Fig. 1 Formation of actinomycin D (IV) from actinomycin halves and formation pathways of 4-methyl-3-hydroxyanthranilic acid (4-MHA) in S. chrysomallus via 4-methyl-3-hydroxykynurenine (MHK) or in S. antibioticus via 3-hydroxyanthranilic acid (3-HA). The asymmetric structure of the phenoxazinone chromophore implies two different sides $(\alpha$ or $\beta$ ) to which the peptide lactone rings can be attached. Moreover the pentapeptide lactone rings can be different (aniso-actinomycins) or be the same (iso-actinomycins) ACMS: actinomycin peptide synthetases, Sar: N-methylglycine, MeVal: N-methyl-L-valine, AdoMet: S-adenosyl-L-methionine, 3-HK: 3-hydroxykynurenine.

(Fig. 1, lower path). ${ }^{\mathbf{1 1}}$ Supporting evidence for this was the earlier finding that 3-HA when added to $S$. antibioticus cultures stimulated actinomycin synthesis apparently through enhanced formation of 4-MHA. ${ }^{12,13}$ However, other in vivo incorporation experiments with $S$. antibioticus showed that externally added 4-MHK stimulated actinomycin formation better than 3-HA which suggested that the methylation step could well be at the stage of 3-HK rather than of 3-HA. ${ }^{14}$

Surprisingly, we found recently that feeding 3-HA to cultures of $S$. chrysomallus or $S$. parvulus had a stimulatory effect on actinomycin synthesis, too. However, analysis of the actinomycin mixtures formed revealed that the increase in product formation exclusively referred to the additional formation of socalled C-demethylactinomycins in which one or both 4-MHA residues in the chromophore were replaced by $3-\mathrm{HA} .{ }^{15}$ In fact, 3-HA was not found to be converted to 4-MHA and instead was incorporated directly into actinomycin half molecules by the 4-MHA-incorporating enzyme ACMSI which can accept 3-HA as a structural homologue of 4-MHA. ${ }^{16}$ These data therefore stand in stark contrast to the reported stimulating effect of 3-HA on actinomycin synthesis in $S$. antibioticus,${ }^{12,13}$ because that same 4-MHA incorporating enzyme ACMSI is also present in $S$. antibioticus. ${ }^{17}$ This prompted us to reinvestigate the effects of 3-HA and also 4-MHA on actinomycin biosynthesis in S. antibioticus. It will be shown that feeding 3-HA and surprisingly also 4-MHA to $S$. antibioticus cells suppresses actinomycin synthesis with concomitant formation of new products formed by competitive intervention of these highly reactive $o$-aminophenol-carboxylic acids in different steps of actinomycin synthesis.

\section{Materials and methods}

\section{Chemicals and radiochemicals}

3-Hydroxyanthranilic acid (3-HA) and fluorescence silica gel $60 \mathrm{~F}_{254}$ coated alumina sheets were from Merck (Darmstadt, Germany). Actinomycin D was purchased from Applichem (Darmstadt, Germany). Daunomycin was isolated from cultures of Streptomyces peucetius ATCC 29050 according to ${ }^{18}$ and purified by HPLC. 3-Hydroxy-4-methylanthranilic acid (4-methyl-3hydroxyanthranilic acid, 4-MHA) was synthesized as described. ${ }^{19}$ $\left[{ }^{14} \mathrm{C}\right.$-methyl $]$-L-methionine (55 $\mathrm{mCi}$ per $\left.\mathrm{mmol}\right)$, U- $\left[{ }^{14} \mathrm{C}\right]-\mathrm{L}-\mathrm{valine}$ (260 mCi per mmol),U-[ $\left.{ }^{14} \mathrm{C}\right]-\mathrm{L}-$ threonine (192 mCi per mmol), U$\left[{ }^{14} \mathrm{C}\right]$-L-proline (252 $\mathrm{mCi}$ per $\left.\mathrm{mmol}\right), \mathrm{U}-\left[{ }^{14} \mathrm{C}\right]$-L-glycine (105 mCi per mmol), U- $\left[{ }^{14} \mathrm{C}\right]$-glutamic acid $\left(238 \mathrm{mCi}\right.$ per mmol) and $\mathrm{L}-\left[5^{-}{ }^{3} \mathrm{H}\right]$ tryptophan (17.9 Ci per mmol) were from Amersham Bioscience (Braunschweig, Germany). All other chemicals were of the highest purity commercially available. Cinnabarinic acid and actinocin were prepared from 3-HA and 4-MHA according to. ${ }^{20}$ The compounds were confirmed by mass spectrometry showing $[\mathrm{M}+$ $\mathrm{H}]^{+}=329$ for actinocin and $[\mathrm{M}+\mathrm{H}]^{+}=301$ cinnabarinic acid.

\section{Strains and cultures}

Strain Streptomyces antibioticus ATCC 14888 (IMRU 3720) was from the American Type Culture Collection (Bethesda, Maryland, USA). It was maintained and grown in the glutamatemineral salts medium supplemented with $1 \%$ galactose plus $0.1 \%$ glucose as described. ${ }^{21}$ Incubation was at $30{ }^{\circ} \mathrm{C}$ and 220 rpm in a New Brunswick G 25 shaker.

\section{Analytical methods}

Solvent systems for thin layer chromatographic separation of actinomycins and hemi-actinomycins was solvent system I (ethylacetate-methanol-water-dimethylformamide, $100: 5: 5: 2$, by vol.). For separation of amino acid mixtures solvent system II (butanol-acetic acid-water; $4: 1: 1$, by vol.) was used. HPLC separations of actinomycin mixtures were performed on a prepacked EnCaPharm 100-RP18 column (Molnar Institute, Berlin, Germany) isocratically with 60\% (by vol.) acetonitrile-water, $0.1 \%$ trifluoroacetic acid (TFA) at a flow rate of $1 \mathrm{ml} \mathrm{min}^{-1}$ using a Pharmacia LKB (Pump 2248, WWM 2141) HPLC system. For isocratic separation of hemiactinomycins, a $52 \%$ (by vol.) acetonitrile-water mixture containing $0.1 \%$ TFA was used. Detection wavelength was $441 \mathrm{~nm}$. 
Actinomycin contents of cultures were determined by extraction of culture aliquots with ethylacetate and subsequent spectrophotometric determination at $441 \mathrm{~nm}$ using authentic actinomycin D as standard. Extracts containing radioactive actinomycins and related compounds were determined by counting aliquots in a Wallac 1409 liquid scintillation counter (Wallac, Turku, Finland). Radioactive compounds on TLC plates were localized by radioscanning with a RITA radioscanner (Raytest, Straubenhardt, Germany) or by autoradiography on Kodak BioMax MR Film. For product analysis, the radioactive zones were scraped off the plates and extracted with methanol. After evaporation to dryness, the material was hydrolyzed in $250 \mu \mathrm{l} 6 \mathrm{M} \mathrm{HCl}$ for $20 \mathrm{~h}$ at $105^{\circ} \mathrm{C}$. The hydrolysate was brought to dryness under reduced pressure and finally dissolved in $50 \%$ ethanol, applied to silica gel plates and chromatographed in solvent system II along with amino acid standards. Radioactive amino acids were detected by radioscanning or autoradiography. Standards were visualized by spraying with $0.1 \%$ ninhydrin in ethanol.

\section{Short term radioincorporation experiments and micro- preparative incubations of streptomycete mycelial suspensions}

Short term radioincorporation experiments were performed as described in. ${ }^{15}$ 3-HA or 4-MHA $(100-250 \mu \mathrm{M})$ were added to mycelial suspensions in the indicated concentrations together with $1 \mu \mathrm{Ci}$ of ${ }^{14} \mathrm{C}$-labeled amino acid, i.e. either threonine, valine, proline, glycine, methionine or $10 \mu \mathrm{Ci}^{3} \mathrm{H}$-labelled tryptophan. Incubation was at $30{ }^{\circ} \mathrm{C}$ and $220 \mathrm{rpm}$ in a New Brunswick G 25 Shaker $(2.5 \mathrm{~cm}$ displacement) for up to $60 \mathrm{~min}$. Radioactive actinomycins and other neutral compounds were extracted twice with $3 \mathrm{ml}$ portions of ethylacetate. $200 \mu \mathrm{l}$ from the combined extracts were counted. The remainder was evaporated to dryness, dissolved in a minute amount of ethylacetate, applied to silica TLC plates and separated by using solvent system I. Micro-preparative incubations supplemented with 3-HA or 4-MHA and the different amino acids of the actinomycin pentapeptide lactone rings were performed as described..$^{15}$ After shaking at $220 \mathrm{rpm}$ and $30{ }^{\circ} \mathrm{C}$ for up to $10 \mathrm{~h}$ each flask was extracted three times with $2 \mathrm{ml}$ portions of ethylacetate. Combined extracts were evaporated to dryness and separated by TLC. Individual bands were scraped off the plates, and subjected to HPLC as described above. Product peaks of interest were lyophilized and subjected to analysis by degradation and/or mass spectrometry.

\section{Alkaline hydrogen peroxide oxidation of actinomycins}

Alkaline oxidative cleavage of actinomycins, hemi-actinomycins, C-demethyl-hemi-actinomycins or C-demethylactinomycins was performed according to. ${ }^{22}$ To the actinomycin or hemi-actinomycin sample (20 to $100 \mu \mathrm{g}$ ) dissolved in $100 \mu \mathrm{l}$ methanol were added $500 \mu \mathrm{l}$ of $3 \%$ hydrogen peroxide in $0.8 \mathrm{~N} \mathrm{NaOH}$. After standing for $30 \mathrm{~min}$ at RT the mixture was acidified to $\mathrm{pH} 2.8$ with diluted $\mathrm{HCl}$ (1.5 $\mathrm{ml}$ final volume) and extracted three times with $1.5 \mathrm{ml}$ portions of ethylacetate. Combined extracts were evaporated to dryness under reduced pressure, separated by HPLC and subjected to HPLC-ESI mass spectrometry.

\section{DNA-binding assays}

Electromobility shift assays (EMSA) were performed in a total volume of $20 \mu \mathrm{l}$ in Tris-buffer $(50 \mathrm{mM})$ at $\mathrm{pH}$ 7.4. The incubations contained $60 \mathrm{ng} \mu^{-1}$ pQE30 plasmid DNA (Qiagen, Hilden, Germany) and $25 \mu \mathrm{g} \mathrm{ml^{-1 }}$ actinomycin D or hemi-actinomycin IV. Controls contained no actinomycin or hemi-actinomycin. After 60 minutes at $37^{\circ} \mathrm{C}$ the incubations were loaded on a $1 \%$ agarose gel $(12 \times 8.4 \mathrm{~mm}$ thickness, TBE buffer $)$ and run at $6 \mathrm{~V} \mathrm{~cm}^{-1}$. After this, bands were visualized by soaking the gel in an ethidium bromide bath for $30 \mathrm{~min}$ and illumination with ultraviolet light.

\section{Topoisomerase I and DNA-gyrase inhibition assay}

Topoisomerase I assay was in a total volume of $20 \mu$ topoisomerase I buffer and in the presence of $100 \mu \mathrm{g}$ bovine serum albumin (protocol by NEB, Frankfurt, Germany). It contained 60 ng pQE30 plasmid DNA (Qiagen, Hilden, Germany), 1 unit topoisomerase I (NEB) and $0.5 \mu \mathrm{g} \mathrm{ml}{ }^{-1}$ actinomycin D or hemiactinomycin IV. The mixtures were allowed to stand at $37^{\circ} \mathrm{C}$ for 2 hours and afterwards were incubated for 15 minutes at $65{ }^{\circ} \mathrm{C}$ for inactivation of topoisomerase I. DNA-gyrase assay was in a total volume of $20 \mu \mathrm{l}$ DNA-gyrase buffer (protocol by NEB, Frankfurt, Germany). The incubations contained $100 \mathrm{ng}$ relaxed pUC19 plasmid DNA (NEB), 1 unit DNA-gyrase (NEB) and $10 \mu \mathrm{g} \mathrm{ml}$ actinomycin $\mathrm{D}$, hemi-actinomycin IV or daunomycin. The mixtures were allowed to stand at $37{ }^{\circ} \mathrm{C}$ for 2 hours and afterwards were incubated for 15 minutes at $65{ }^{\circ} \mathrm{C}$ for inactivation of DNA-gyrase. Electrophoretic separation was performed on a $1 \%$ agarose gel as above. Staining of bands was with ethidium bromide.

\section{Inhibition assays}

Disc diffusion assays for testing antimicrobial activity of hemiactinomycin and actinomycin against Bacillus subtilis ATCC 6633 were performed by standard procedures. Defined amounts of compounds (dissolved in ethanol) were spotted on paper discs (6 mm diameter, Schleicher and Schuell) and after drying were laid on a soft top nutrient agar (Difco) inoculated with $0.1 \%$ (v/v) of an overnight culture of Bacillus subtilis ATCC 6633. After incubation at $30{ }^{\circ} \mathrm{C}$ over night diameters of inhibition zones were determined.

\section{Mass spectrometric analysis}

MALDI-TOF mass spectra were recorded using a Bruker Autoflex instrument equipped with a $337 \mathrm{~nm}$ nitrogen laser for desorption and ionization. $2 \mu \mathrm{l}$ samples of HPLC-purified actinomycin species were mixed with the same volume of matrix medium (a saturated solution of $\alpha$-cyano-4-hydroxycinnamic acid in $50 \%$ aqueous acetonitrile containing $0.1 \%$ trifluoroacetic acid), spotted on the target, air dried and measured. Spectra were recorded by positive ion detection in reflector mode. The acceleration and reflector voltages were 19 and $20 \mathrm{kV}$ in pulsed ion extraction mode. A molecular gate of 350 Da improved the measurement by filtering out most matrix ions. Monoisotopic mass numbers were obtained. The structure of the new actinomycins was investigated by post source decay (PSD)-MALDI- 
TOF mass spectrometry. The fragment spectra of C-demethylactinomycins, hemi-actinomycins and C-demethyl-hemi-actinomycins were compared with that of actinomycin as the reference.

HPLC-ESI-MS experiments were recorded using an Exactive ESI-Orbitrap-MS (Thermo Fisher Scientific GmbH, Bremen, Germany) that was coupled to a HPLC 1200 Agilentsystem (Agilent, Waldbronn, Germany). The scans were performed as full scans $(100-2000 \mathrm{~m} / \mathrm{z}$ or $100-1000 \mathrm{~m} / \mathrm{z})$ and recorded by positive and negative ion detection ( 2 scan events). HPLC was performed using a $50 \times 2 \mathrm{~mm}$ GRACE $3 \mu$ Grom-Sil ODS-4 120 column (Grace Davison, Deerfield, USA) with a flow rate of

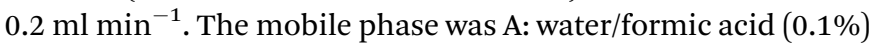
and B: acetonitrile/formic acid (0.1\%). A linear gradient was used from 5 to $100 \%$ B over $10 \mathrm{~min}$, after which it was switched in $0.1 \mathrm{~min}$ to $5 \% \mathrm{~B}$ and remained there for $5 \mathrm{~min}$.

\section{Results and discussion}

\section{3-HA induces synthesis of novel actinomycins in Streptomyces antibioticus}

S. antibioticus IMRU 3720 produces actinomycins IV and V as main components of the actinomycin $\mathrm{X} \operatorname{complex}^{23}$ (Fig. S1†). Actinomycin IV (synonymous with actinomycin D in Fig. 1) is an iso-actinomycin possessing two identical pentapeptide lactone rings each containing proline in the 'proline' site whereas actinomycin $\mathrm{V}$ is an aniso-actinomycin with 4-oxoproline instead of proline in its $\beta$-pentapeptide lactone ring. There is also actinomycin I, a minor product, where the $\beta$-ring contains a 4-hydroxyproline residue in the 'proline'-position ${ }^{23}$ (Fig S1†).

To examine the effect of 3-HA on actinomycin synthesis in $S$. antibioticus, washed mycelium was incubated in short-term radioincorporation experiments with ${ }^{14} \mathrm{C}$-threonine and $250 \mu \mathrm{M}$ 3-HA. Fig. 2 (lane 2) shows the separation of the ethylacetateextractable products which reveals that addition of 3-HA had led to the formation of a variety of new labeled bands. Concomitantly, the intensities of the actinomycin IV and V bands were less intense when compared to the control experiment without 3-HA (Fig. 2, lane 1), indicating that the new compounds had been formed at the expense of actinomycins. Recently obtained knowledge of C-demethylactinomycins from $S$. chrysomallus and $S$. parvulus suggested that the new compounds shortly moving under the bands of actinomycin IV or V could be their corresponding C-demethylactinomycins. ${ }^{15}$

These were the indicated double band ( $\mathrm{D}_{\mathrm{IV}}$ and $\left.\mathrm{DD}_{\mathrm{IV}}\right)$ under the band of actinomycin IV $\left(R_{\mathrm{f}} \sim 0.5\right)$ and two distinct bands $\left(\mathrm{D}_{\mathrm{V}}(1)\right.$ and $\left.\mathrm{D}_{\mathrm{V}}(2)\right)$ under the band of actinomycin $\mathrm{V}\left(R_{\mathrm{f}} \sim 0.66\right)$. However, there were also two further prominent bands with lower $R_{\mathrm{f}} \mathrm{s}$, designated $\mathrm{DH}_{\mathrm{IV}}$ and $\mathrm{DH}_{\mathrm{V}}$ for which no counterpart were seen to be formed in short term incubations of $S$. chrysomallus and S. parvulus ${ }^{\mathbf{1 5}}$ and therefore most likely were no C-demethylactinomycins.

Interestingly, the analysis of parallel incubations where cells of $S$. antibioticus had been incubated with externally added 4MHA instead of 3-HA revealed that 4-MHA suppressed actinomycin synthesis also with concomitant formation of two new compounds, $\mathrm{H}_{\mathrm{IV}}$ and $\mathrm{H}_{\mathrm{V}}$, having coordinately higher $R_{\mathrm{f}}$-values

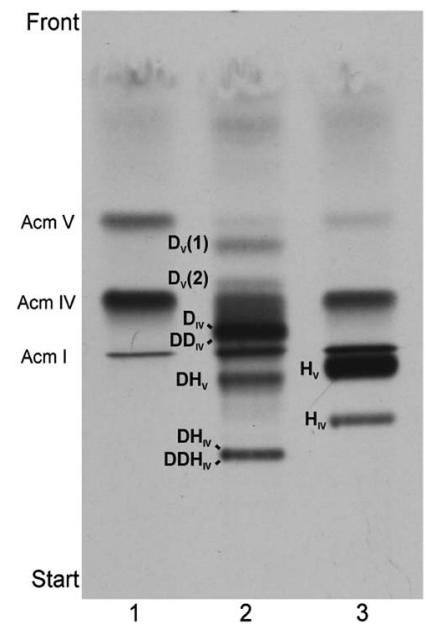

Fig. 2 Formation of C-demethylactinomycins and hemi-actinomycins in S. antibioticus. Lane 1: separation of actinomycin mixture formed in mycelial suspensions (with labeling by ${ }^{14} \mathrm{C}$-threonine). Lane 2: separation of actinomycins formed in the presence of $250 \mu \mathrm{M} 3-\mathrm{HA}$. Lane 3: separation of actinomycins formed in the presence of $250 \mu \mathrm{M}$ 4-MHA. Separation was on silica thin-layer plates using solvent system I. Time of exposure of the chromatogram to X-ray film was 2 days. Acm: actinomycin. The abbreviations used to indicate the different new bands formed upon addition of 3-HA or 4-MHA are explained in the text.

than the 3-HA-inducible ones. This suggested, that they were the homologues of compounds $\mathrm{DH}_{\mathrm{IV}}$ and $\mathrm{DH}_{\mathrm{V}}$ and contained 4MHA instead of 3-HA. Radiochemical analysis of all new compounds by acid hydrolysis and amino acid analysis revealed that they contained ${ }^{14} \mathrm{C}$-threonine. Similarly, ${ }^{14} \mathrm{C}$-valine and ${ }^{14} \mathrm{C}-\mathrm{N}$-methyl-L-valine were recovered from the compounds when ${ }^{14} \mathrm{C}$-valine was used in parallel experiments to label these bands (Fig. S2 $\dagger$ ). These data indicated that the 3-HA or 4-MHAinducible new compounds possessed the same peptide portions as their corresponding actinomycins.

Larger non-labeled quantities of these compounds were produced in micro-preparative incubations in the presence of $250 \mu \mathrm{M} 3-\mathrm{HA}$ (or 4-MHA) and $2 \mathrm{mM}$ of the amino acids of the pentapeptide lactone rings (see Materials and methods). The separation of extracts from these incubations by preparative TLC revealed an identical pattern of new bands formed in the presence of the two aminophenol carboxylic acids like in the short-term incubations with radiolabeled amino acids. All new bands had a red-orange or brownish-orange colour indicating that they contained a phenoxazinone chromophore (Fig. S3 $\dagger$ ). After isolation from plates, each compound was purified further by HPLC. Based on their spectral absorption at $441 \mathrm{~nm}$ and taking the published extinction coefficient for actinomycin D, the typical total yield of new compounds from a micro-preparative incubation was between 50 and $150 \mu \mathrm{g}\left(8-20 \mu \mathrm{g} \mathrm{ml} \mathrm{m}^{-1}\right)$.

\section{Identification of C-demethylactinomycins from $S$. antibioticus}

For clarification of the various 3-HA- or 4-MHA-inducible compounds we first turned to the presumptive C-demethylactinomycins. Mass spectrometry revealed that compound 
$\mathrm{D}_{\text {IV }}$ had a mass peak of $[\mathrm{M}+\mathrm{H}]^{+}=1242$ and by comparison with the earlier confirmed same compound from $S$. parvulus ${ }^{15}$ was identified as mono-( $\alpha)$-C-demethylactinomycin IV (D) (Fig. S1 $\dagger$ ). The accompanying compound DD $_{\text {IV }}$ showed a mass peak $[\mathrm{M}+\mathrm{H}]^{+}=1228$ in agreement with the structure of di-C-demethylactinomycin IV (Fig. S1 $\dagger$ ). Compounds $\mathrm{D}_{\mathrm{V}}(1)$ and $\mathrm{D}_{\mathrm{V}}(2)$ surprisingly showed the same mass peak of $[\mathrm{M}+\mathrm{H}]^{+}=1255$ which indicated for both a molecular weight consistent with that of a mono-C-demethylactinomycin $\mathrm{V}$ for which four isomeric structures are theoretically possible (Fig. $\mathrm{S} 1 \dagger$ ).

To determine the location of the 3-HA residue in the phenoxazinone chromophores of these C-demethylactinomycins, in vivo biosynthetic labeling experiments with $5-{ }^{3} \mathrm{H}$-tryptophan in the presence of external 3-HA was performed (Fig. 3a). Taking into account that the $5-{ }^{3} \mathrm{H}$-tryptophan only labels intracellularly formed 4-MHA and that 3-HA remains unlabeled ${ }^{\mathbf{1 5 , 2 4}}$ two possibilities exist for the fate of the tritium in the 5-position of labeled 4-MHA (Fig. 3b). When 4-MHA is on the $\alpha$-side of the phenoxazinone moiety, the label of radioactive 4-MHA is retained, however, is lost when on the $\beta$-side (Fig. 3b). The data in Fig. 3a (lane 2 and 3) show that 5 - ${ }^{3} \mathrm{H}$-tryptophan labels only $\mathrm{D}_{\mathrm{V}}(2)$ whereas compound $\mathrm{D}_{\mathrm{V}}(1)$ and also $\mathrm{D}_{\mathrm{IV}}$ remain unlabeled. This shows that in both $\mathrm{D}_{\mathrm{V}}(1)$ and $\mathrm{D}_{\mathrm{IV}}$ the 3-HA moiety was incorporated into the $\alpha$-side and thus are mono- $(\alpha)$-C-

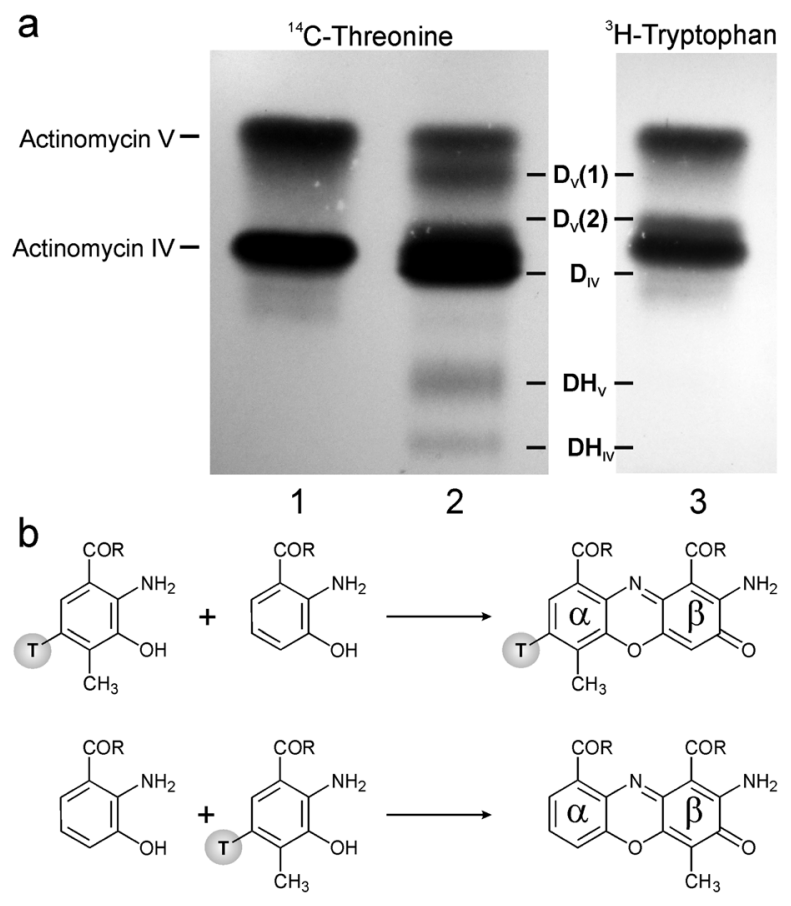

Fig. 3 Regioselective incorporation of 3-HA into C-demethylactinomycin V(1) and V(2). (a) Lanes $1:{ }^{14} \mathrm{C}$-threonine-labeled actinomycin IV and $V$ formed in mycelial suspension of $S$. antibioticus. Lane $2:{ }^{14} \mathrm{C}$ threonine-labeled actinomycins, C-demethylactinomycins and Cdemethyl-hemi-actinomycins formed in the presence of 3-HA. Lane 3: actinomycins, C-demethylactinomycins and C-demethyl-hemiactinomycins after labeling with $5-{ }^{3} \mathrm{H}$-tryptophan formed in the presence of 3-HA. (b) Scheme of biosynthetic labeling of $D_{V}(1)$ and $\mathrm{D}_{\vee}(2)$ with $5-{ }^{3} \mathrm{H}$-tryptophan. $\mathrm{R}$ : pentapeptide lactone rings. demethylactinomycins. In contrast, $\mathrm{D}_{\mathrm{v}}(2)$ is a mono- $(\beta)-\mathrm{C}-$ demethylactinomycin. Formation of a mono-( $\beta)$-C-demethylactinomycin such as $\mathrm{D}_{\mathrm{v}}(2)$ has not been detected in $S$. parvulus when fed with 3-HA. There, only mono-( $\alpha$ )-C-demethylactinomycin IV $\left(\mathrm{D}_{\mathrm{IV}}\right)$ was formed. ${ }^{15}$ However, the latter is an iso-actinomycin with two identical pentapeptide lactone rings whereas mono-C-demethylactinomycins $\mathrm{V}$ are aniso-actinomycins containing two non-identical pentapeptide lactone rings. Apparently, the presence of different pentapeptide lactone rings has influence on the regiospecificity of the phenoxazinone formation reaction in $S$. antibioticus. It would be interesting, therefore, to know the location of the 4-oxo-proline containing pentapeptide lactone ring in compounds $\mathrm{D}_{\mathrm{V}}(1)$ and $\mathrm{D}_{\mathrm{V}}(2)$. Due to the limited amounts of available material, its localization has not been achieved, yet.

\section{3-HA and 4-MHA induce synthesis of hemi-actinomycins}

The other two pairs of 3-HA- $\left(\mathrm{DH}_{\mathrm{IV}}\right.$ and $\left.\mathrm{DH}_{\mathrm{V}}\right)$ and 4-MHAinducible $\left(\mathrm{H}_{\mathrm{V}}\right.$ and $\left.\mathrm{H}_{\mathrm{IV}}\right)$ compounds on TLC-plates showed a brownish-orange colour which distinguished them from the red-orange coloured actinomycins (Fig. S3†). In methanolic solution, their absorption maxima were different from that of actinomycin D (445 nm vs. $441 \mathrm{~nm}$ ) and the compounds showed a magenta-like colour in which they more resembled the magenta colour of the actinomycin chromophore actinocin $\left(\lambda_{\max }=451 \mathrm{~nm}\right)$. We argued therefore, that they represented phenoxazinones carrying only one pentapeptide lactone ring and a free carboxy group which could be formed by the oxidative condensation of abundant 4-MHA or 3-HA with actinomycin halves (Fig. 4).

MALDI-TOF mass spectrometry of the anticipated hemiactinomycins $\left(\mathrm{H}_{\mathrm{IV}}\right.$ and $\left.\mathrm{H}_{\mathrm{V}}\right)$ and $\mathrm{C}$-demethyl-hemi-actinomycins $\left(\mathrm{DH}_{\mathrm{IV}}\right.$ and $\left.\mathrm{DH}_{\mathrm{V}}\right)$ confirmed this assumption. Compound $\mathrm{H}_{\mathrm{IV}}$ displayed a mass peak $[\mathrm{M}+\mathrm{H}]^{+}$at $m / z=792.5$ in accordance with the proposed structure as hemi-actinomycin IV (Fig. 5a). Compound $\mathrm{H}_{\mathrm{V}}$ had a mass peak $[\mathrm{M}+\mathrm{H}]^{+}$at $m / z=806.6$ in agreement with the proposed structure of hemi-actinomycin $\mathrm{V}$ (Fig. 5b). These conclusions were further corroborated by PSDMALDI-TOF mass spectrometric analysis for each compound which revealed the same fragmentation pattern for the peptide lactone rings as for actinomycin IV or V (Fig. S5 and Table S1 $\dagger$ ). For the anticipated mono-C-demethyl-hemi-actinomycin $\mathrm{DH}_{\mathrm{IV}}$ a

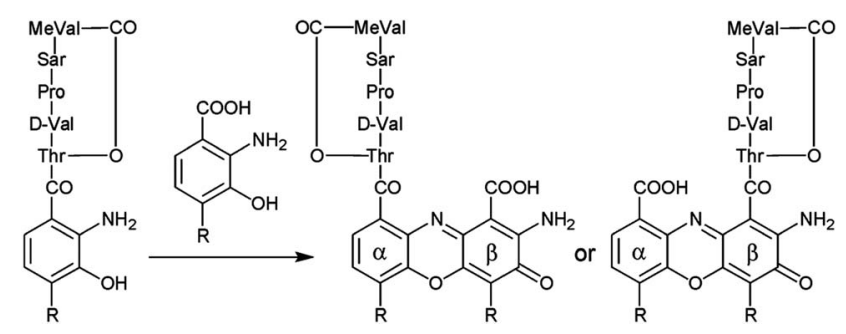

Fig. 4 Structures of hemi-actinomycins formed in the presence of excess 3-HA or 4-MHA. Schematic formation of hemi-structured actinomycins in S. antibioticus in the presence of abundant 3-HA or 4MHA. R: methyl or $\mathrm{H}$. In case of demethyl-hemi-actinomycins the $\mathrm{R}$ substituent my be variable between the two possibilities. 

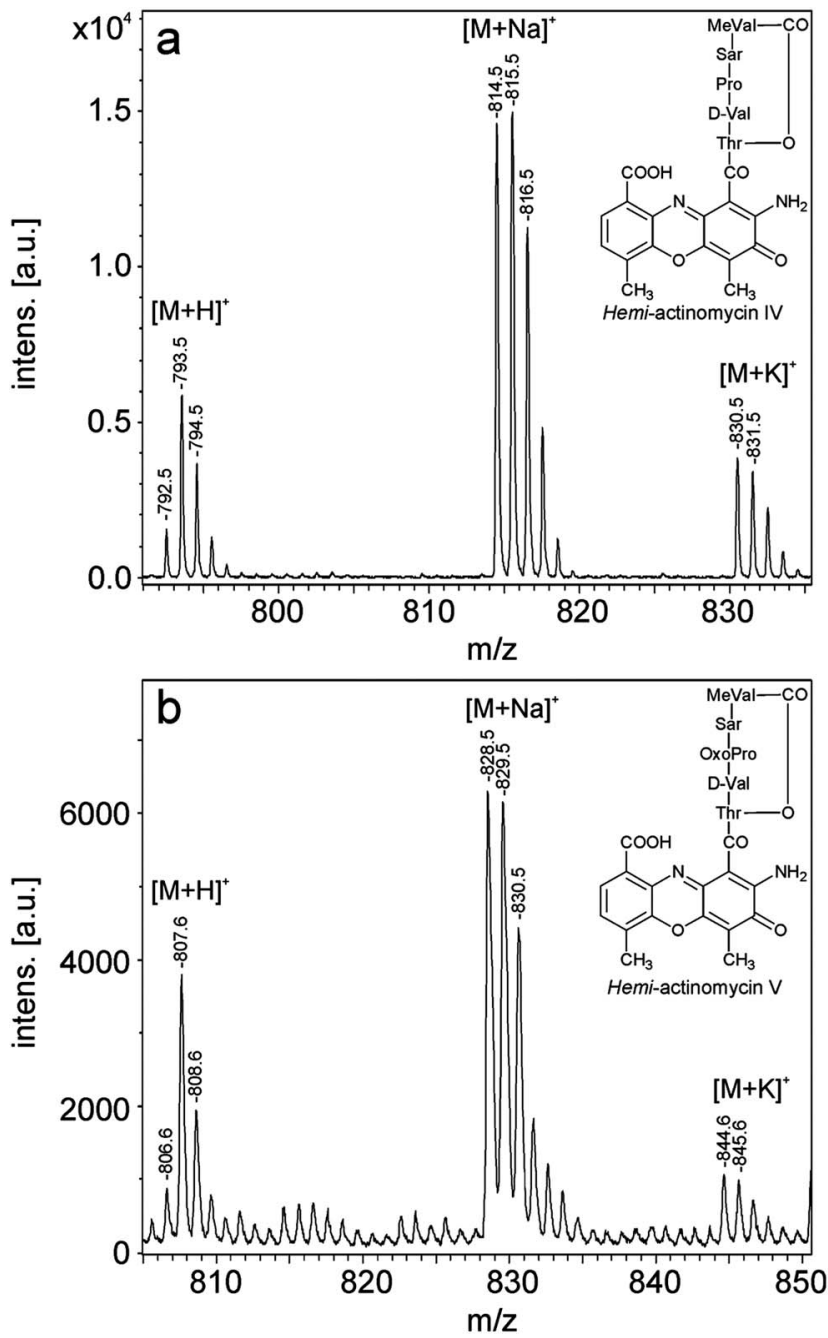

Fig. 5 MALDI-TOF mass spectra of hemi-actinomycin IV (a) and V (b). (a) Spectrum of hemi-actinomycin IV $[\mathrm{M}+\mathrm{H}]^{+} \mathrm{m} / z=792$. (b) Spectrum of hemi-actinomycin $\mathrm{V}[\mathrm{M}+\mathrm{H}]^{+} \mathrm{m} / \mathrm{z}=806$.

mass peak $[\mathrm{M}+\mathrm{H}]^{+}$of $m / z=778.5$ verified its identity. Interestingly, the compound was accompanied by small amounts of a sister compound displaying an $[\mathrm{M}+\mathrm{H}]^{+}$of $m / z 764.4$, most probably representing the di-demethyl derivative of hemi-actinomycin IV (Fig S4†). Their assignment was again confirmed by fragment analysis applying PSD-MALDI-TOF mass spectrometry (Fig S5 and Table S1 $\dagger$ ). The mass data of representative fragment ions are listed in Table $\mathrm{S} 1 \dagger$ covering both fragments of the peptide lactone ring and those which originate by elimination of peptide portions of both demethylated species. Corresponding fragments of the mono-C-demethyl- and di-Cdemethyl derivatives appeared at 14 and 28 mass units smaller than those of hemi-actinomycin IV lacking one and two methyl groups at the phenoxazinone chromophore, respectively. Their names, masses and $R_{\mathrm{f}}$ values are listed in Table $\mathrm{S} 2 . \dagger$

To determine at which sides ( $\alpha$ - or $\beta$-) of the C-demethylhemi-actinomycins the 3-HA residues were located, in vivo biosynthetic labeling of compounds $\mathrm{D}_{\mathrm{V}}(1)$ and $\mathrm{D}_{\mathrm{V}}(2)$ with $5{ }^{-}{ }^{3} \mathrm{H}-$ tryptophan in the presence of external 3-HA was performed. The TLC data in Fig. 3 (lower part of chromatogram) clearly show that though both mono-C-demethyl-hemi-actinomycins $\left(\mathrm{DH}_{\mathrm{IV}}\right.$ and $\mathrm{DH}_{\mathrm{V}}$ ) were labeled with threonine (lane 2) no label derived from $5-{ }^{3} \mathrm{H}$-tryptophan was detectable (lane 3 ). This indicated that the tritium of 4-MHA was lost during phenoxazinone formation which left no other possibility that in both compounds the 3-HA residue was incorporated into the $\alpha$-side. Furthermore, to determine on which side of hemi-structured actinomycins (and their demethyl-homologs) the pentapeptide lactone rings are located we degraded hemi-actinomycin IV (compound $\mathrm{H}_{\mathrm{IV}}$ ) by treatment with alkaline hydrogenperoxide. This cleaves actinomycins into two different linear pentapeptide derivatives, one derived from the $\alpha$-side with 7-methylbenzoxazalone-4-carboxylic acid at its amino terminus and the other from the $\beta$-side carrying oxalic acid 22 (Fig. S6 $\dagger$ ).

Fig. 6 shows the mass spectrum of products obtained by $\mathrm{NaOH} / \mathrm{H}_{2} \mathrm{O}_{2}$-cleavage of hemi-actinomycin IV $\left(\mathrm{H}_{\mathrm{IV}}\right)$ and - for comparison - that of actinomycin IV. While in case of cleavage of actinomycin IV the 7-methylbenzoxazalone-4-carbonyl- as well as the oxalyl-pentapeptide were obtained (Fig. 6, upper part) the cleavage of hemi-actinomycin exclusively delivered the oxalyl-pentapeptide. This clearly proves that the single pentapeptide lactone ring of the hemi-actinomycins is located to the $\beta$-side of the phenoxazinone residue. Obviously, the condensation reaction between free 3-HA or free 4-MHA with 4-MHA(or 3-HA-) pentapeptide lactone rings was regiospecific. The known chemistries of phenoxazinone formation catalysed by e.g. peroxyl radicals ${ }^{25}$ or by enzymes such as tyrosinase $(\mathrm{GriF})^{\mathbf{2 6}}$ or phenoxazinone synthase (PHS) ${ }^{27}$ imply as first step a 2-electron withdrawal from the $o$-aminophenol creating a quinone imine which in turn is attacked by the amino group of a second $o$-aminophenol (Fig. 7a). In case of unsymmetrical phenoxazinones like grixazone from $S$. griseus, the first step of phenoxazinone condensation is the oxidation of 2 - $(\mathrm{N}$-acetylcysteaminyl)-3-amino-4-hydroxybenzaldehyde (2-NAC-3,4AHBAL) to the corresponding quinone imine which in turn reacts with 3,4-AHBAL to grixazone with incorporation of the bulkier 2-NAC-3,4-AHBAL to the $\beta$-side of the phenoxazinone (Fig. 7b). The final phenoxazinones are then formed in two further 2-electron removing steps (omitted in Fig. 7b). The regiochemistry of grixazone therefore reflects in a way the formation of the $\beta$-oriented hemi-actinomycins suggesting that the aminophenol carrying a larger substituent ortho to the amino group is faster converted to the quinone imine than the non-substituted one. Similar findings have been reported on the formation of different unsymmetrical phenoxazinones catalysed by laccase. ${ }^{28}$

Apparently, in S. antibioticus the formation of hemi-actinomycins is similarly regiospecific directing the actinomycin half molecule to the $\beta$-side of the phenoxazinone nucleus although both 3-HA or 4-MHA - when externally added - are in excess over the concentrations of actinomycin halves in the cells. Nevertheless, during the incubations the symmetrical chromophores cinnabarinic acid or actinocin, respectively, were produced in parallel and in appreciable amounts as revealed by the presence of their bands of $R_{\mathrm{f}} \sim 0.15$ and $\sim 0.19$ both showing a magenta colour on TLC plates (Fig. S3†). The identity of both compounds was confirmed by co-chromatography with authentic references. 


\section{a-side}

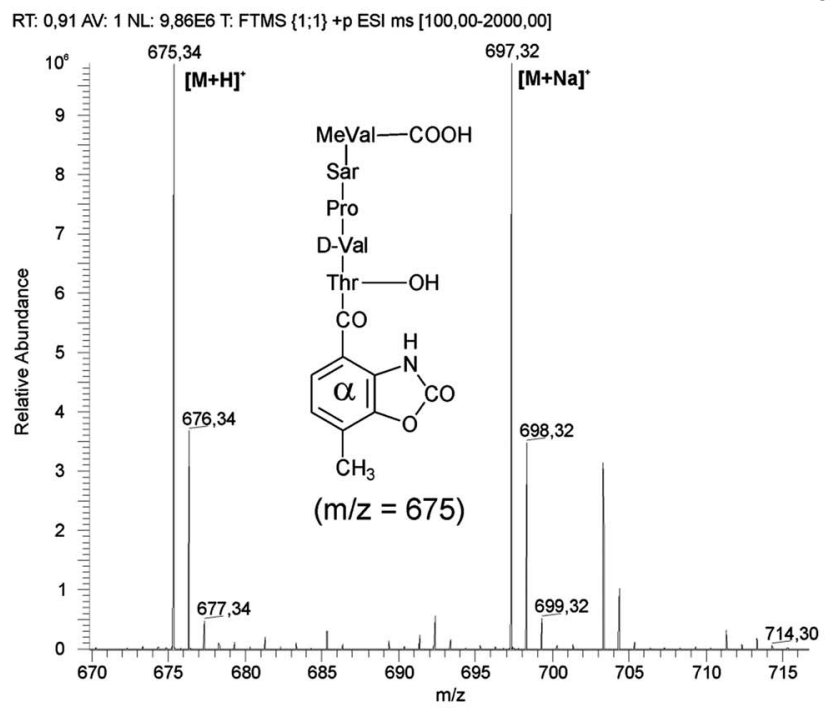

Actinomycin IV $\quad \beta$-side

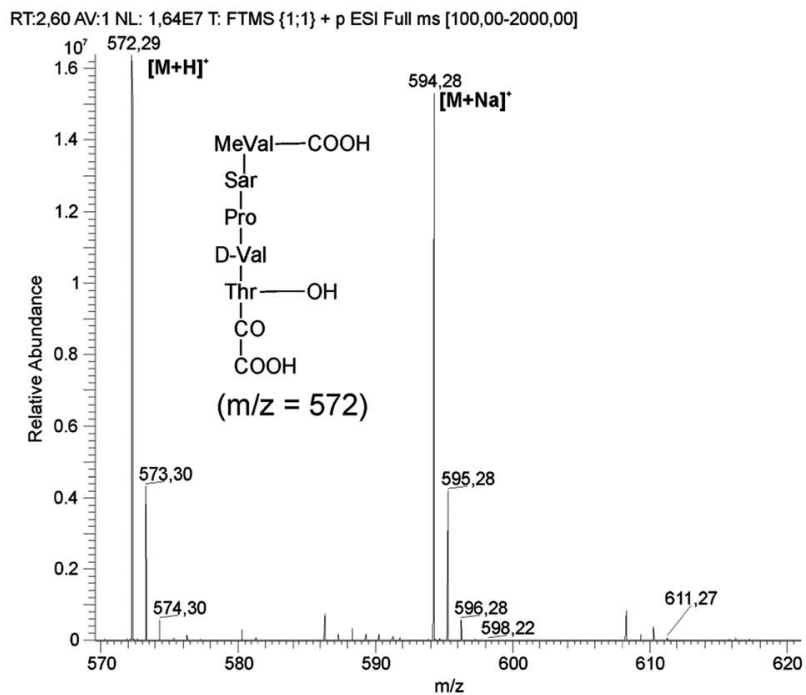

\section{Hemi-Actinomycin IV}
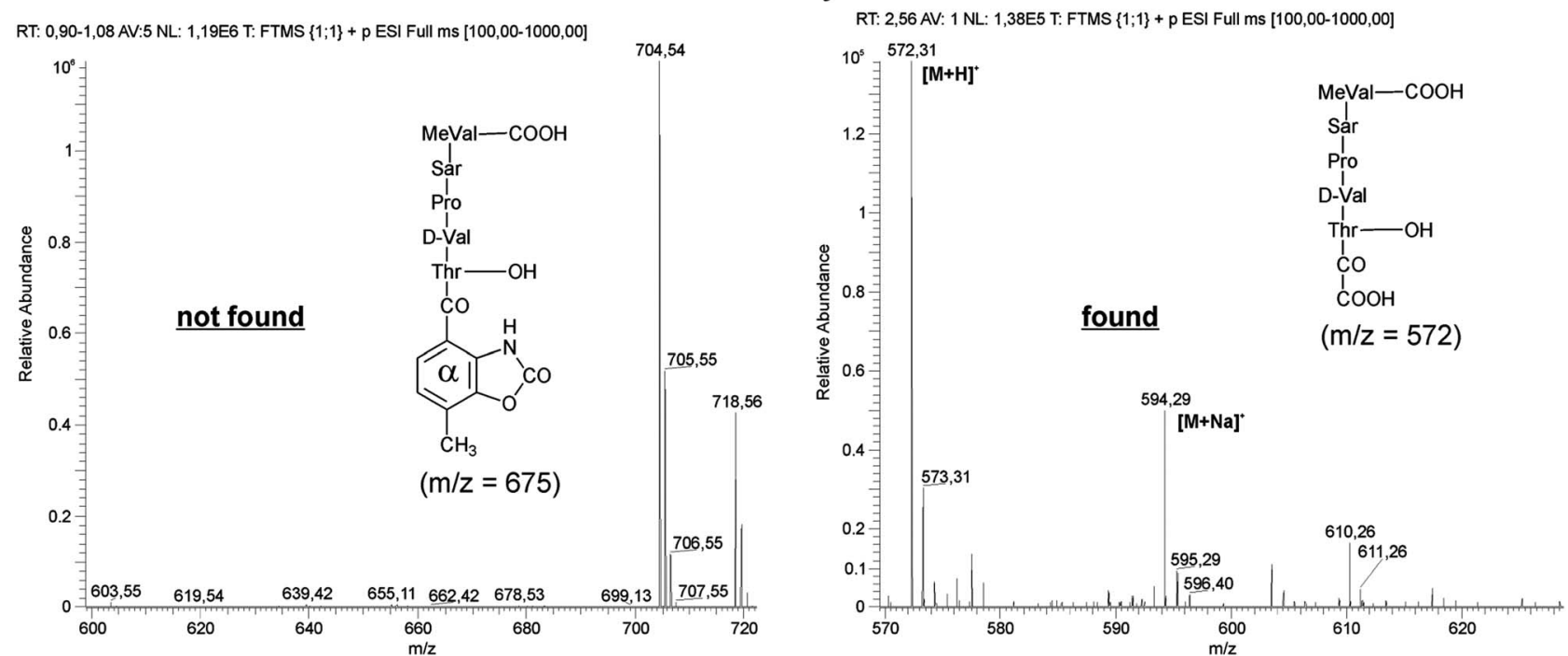

Fig. 6 Mass spectrometric analysis of alkaline hydrogen peroxide digested actinomycin IV and hemi-actinomycin IV. The digest was separated by HPLC and factions containing fragments of cleavage analysed by mass spectrometry. Upper panel: digest of $100 \mu \mathrm{g}$ actinomycin IV contains both fragments of the $\alpha$ - and $\beta$-side. Lower panel: digest of $45 \mu \mathrm{g}$ hemi-actinomycin IV contains only the peptide fragment derived from the $\beta$-side.

In contrast, neither formation of cinnabarinic acid or actinocin was detectable in parallel incubations of mycelial suspensions of S. chrysomallus or S. parvulus with 3-HA or 4-MHA nor was formation of hemi-actinomycins observed. ${ }^{15}$ It is known that S. antibioticus possesses phenoxazinone synthase (PHS) but that this enzyme is missing in $S$. chrysomallus and $S$. parvulus. ${ }^{29}$ The presence of PHS in S. antibioticus was directly visible during the incubations with 3-HA or even stronger with 4-MHA because mycelial suspensions turned deeply red when incubated with these compounds. In contrast, the mycelial suspensions of S. chrysomallus or S. parvulus in the same conditions developed a slight reddish tone only. We checked PHS activity in protein extracts from $S$. antibioticus. These extracts catalysed conversion of 4-MHA to actinocin or 3-HA to cinnabarinic acid. In contrast, protein extracts from $S$. chrysomallus or $S$. parvulus did not possess such enzyme activity.

We also considered the possibility that the hemi-actinomycins and demethyl-hemi-actinomycins were formed from preformed actinocin or demethyl-cinnabarinic acid to which the peptide chain of actinomycin had been added instead by condensation of 4-MHA or 3-HA with actinomycin halves. However, the fact that in the presence of 3 -HA mono- $\alpha-\mathrm{C}$ demethyl-hemi-actinomycin IV $\left(\mathrm{DH}_{\mathrm{IV}}\right)$ was always formed in excess over the di-C-demethyl-hemi-actinomycin IV clearly excludes this possibility. Since in incubations with 3-HA formation of cinnabarinic acid is observed in abundance (Fig. S3†) one had to expect formation of excess di-C-demethylhemi-actinomycins which, however, was not observed. It 
a

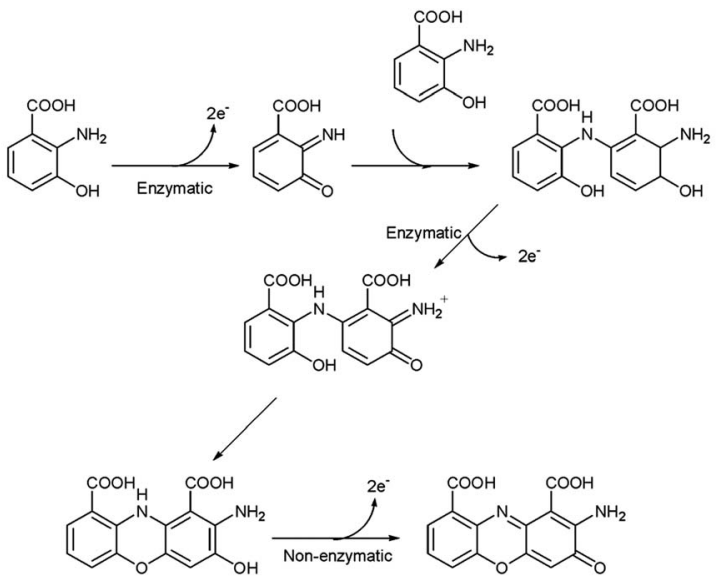

b

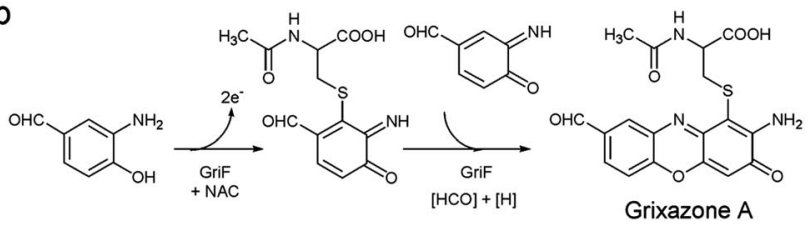

Fig. 7 Schemes of enzymatic phenoxazinone formations. (a) Mechanism of PHS from Streptomyces antibioticus in the oxidation of 3- $\mathrm{HA}^{30}$ (b) mechanism of tyrosinase GriF from Streptomyces griseus catalysing condensation of 3-amino-4-hydroxybenzaldehyde with 2-N-acetyl-cysteinyl-3-amino-4-hydroxybenzaldehyde in the formation of grixazone. ${ }^{26}$

therefore is unlikely that preformed phenoxazinone chromophores are precursors of C-demethyl-hemi-actinomycins or hemi-actinomycins. Thus, the most conceivable route of formation of demethyl-hemi-actinomycins or hemi-actinomycins is via the condensation of 3-HA or 4-MHA with preformed actinomycin halves.

\section{Drug activity of hemi-actinomycin as DNA-intercalator}

Hemi-actinomycin IV was tested for its ability to bind to DNA. Fig. 8a shows electromobility shifts with plasmid DNA in the presence and absence of hemi-actinomycin IV or actinomycin IV. There is a clear shift of plasmid DNA to higher molecular weight in the presence of hemi-actinomycin IV as well as of actinomycin IV. In case of the latter the shift was higher in accordance with the higher molecular weight of actinomycin IV compared with hemi-actinomycin IV (1255 vs. 792). The fact that hemi-actinomycin has a negative charge at neutral $\mathrm{pH}$ may also be a reason. In any case, this finding suggests that hemi-actinomycin IV intercalates into DNA like actinomycin IV. It is known that the intercalation of actinomycin to DNA is through the major groove but that it is stabilized by the two pentapeptide lactone rings extending perpendicularly to the chromophore into both directions of the minor groove. ${ }^{31}$ The data shown here indicate that one ring alone is sufficient for binding of hemi-actinomycin to the minor groove of DNA. In contrast, incubating plasmid DNA with the actinomycin chromophore actinocin did not result in shifts.

Actinomycin is a known inhibitor of topoisomerase I preventing relaxation of cccDNA by stabilizing the open complex a
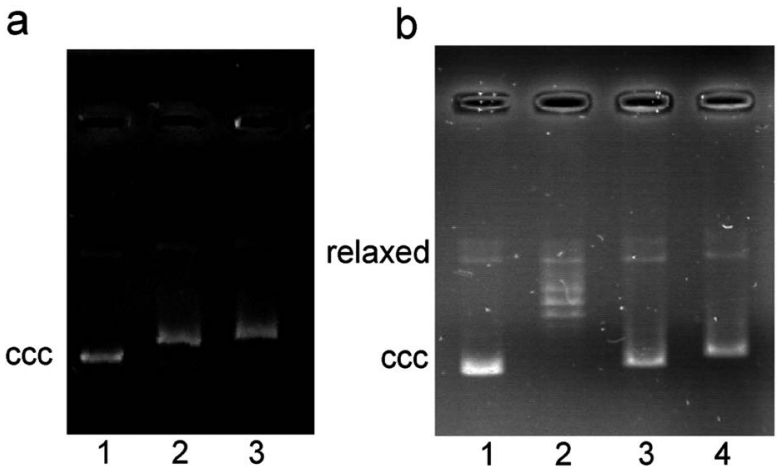

C

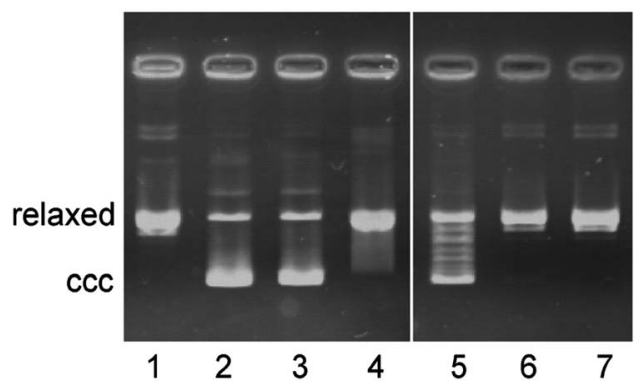

Fig. 8 Hemi-actinomycins bind to DNA and inhibit topoisomerase I and DNA-gyrase. (a) Electromobility shift assay with plasmid PQE30 (ccc form). 1 no addition, 2 with hemi-actinomycin, 3 with actinomycin. (b) Relaxation of plasmid pQE30 (ccc form) by topoisomerase. 1 no addition, 2 with topoisomerase I, 3 with topoisomerase I and hemiactinomycin, 4 with topoisomerase I and actinomycin. (c) Supercoiling of plasmid pUC19 (relaxed form) by DNA gyrase. 1 plasmid only, 2 with gyrase ( $4 \mathrm{~h}$ incubation), 3 with gyrase ( $8 \mathrm{~h}$ incubation), 4 with gyrase and daunomycin ( $2 \mathrm{~h}$ incubation), 5 with gyrase ( $2 \mathrm{~h}$ incubation), 6 with gyrase and hemi-actinomycin, 7 with gyrase and actinomycin, for details see Materials and methods.

between DNA and the enzyme. ${ }^{32}$ Fig. $8 \mathrm{~b}$ shows that topoisomerase I relaxes cccDNA with formation of a ladder of different conformers. As to be expected, in the presence of actinomycin IV no ladder is formed and the plasmid DNA remains ccc. Similarly, also in the presence of hemi-actinomycin IV no ladder is formed.

We were also interested to see whether hemi-actinomycin inhibited DNA gyrase. Relatively little is known about the inhibiting effect of actinomycin on DNA gyrase. Since daunomycin which like actinomycin is a DNA-intercalating drug and strongly inhibits DNA gyrase we expected that actinomycin and eventually hemi-actinomycin would inhibit gyrase also. ${ }^{33}$ Fig. $8 \mathrm{c}$ shows that actinomycin inhibits supercoiling of relaxed DNA by DNA gyrase. Instead of forming the ladder consisting of different topoisomers, the enzyme was inhibited by actinomycin and the DNA stayed relaxed. In agreement with the data obtained with topoisomerase I, hemi-actinomycin IV also inhibited DNA gyrase which strongly indicates that hemi-actinomycin exerted its effect through intercalation into DNA (Fig. 8).

$H e m i$-actinomycin IV was tested for antimicrobial activity against Bacillus subtillis. Plate diffusion tests showed in vivo inhibitory activity of hemi-actinomycin IV in accordance with the inhibitory action of hemi-actinomycin against topoisomerase I 
and DNA gyrase. Semiquantitative determination revealed that hemi-actinomycin was about 15 fold less active than actinomycin (as estimated from diameter of inhibition zones at different concentrations of hemi-actinomycin IV and actinomycin IV) (Fig S7†).

\section{Natural occurrence of hemi-actinomycins}

It is known that the highly reactive 4-MHA is present in the cell of actinomycin-producing streptomycetes at very low concentration. ${ }^{16,19}$ This may be necessary to avoid premature condensation of 4-MHA molecules with each other yielding actinocin or as we know now with actinomycin halves leading to hemi-actinomycins. Nevertheless, we expected that small amounts of 4-MHA could accumulate during longer cultivations of $S$. antibioticus and react with actinomycin halves resulting in formation of hemi-actinomycins. This expectation was based on our earlier observations that long-incubated cultures of S. chrysomallus or $S$. parvulus contain small amounts of C-demethylactinomycins formed from traces of $3-\mathrm{HA}$ that were continuously formed from premature cleavage of 3-hydroxykynurenine that escaped the 4-MHA pathway (Fig. 1). ${ }^{15}$ Mass spectrometric analysis of extracts from such 1 week old cultures of $S$. antibioticus in fact revealed the presence of trace amounts of both hemi-actinomycin V and IV. However, the amounts of these compounds were too low to analyse their regiochemistry.

\section{Conclusions}

That feeding 3-HA or 4-MHA to actinomycin-producing $S$. antibioticus gave rise to the formation of $\beta$-hemi-actinomycins and $\beta$-C-demethyl-hemi-actinomycins, respectively, with suppression of actinomycin shows that these structurally similar aminophenolic carboxylic acids can intervene in the phenoxazinone forming reaction by prematurely condensing with actinomycin halves. The data also show 3-HA is not precursor of 4-MHA because - apart from its role as precursor of demethylactinomycin - 3-HA induces synthesis of mono-C-demethyl-hemi-actinomycins and to lesser extent of di-C-demethyl-hemi-actinomycins but not of hemi-actinomycins. Thus, 3-HA plays only a role as a substrate analogue of 4-MHA in the assembly reaction of actinomycin halves or during phenoxazinone formation snatching away actinomycin halves before they condense with another actinomycin half giving actinomycin or demethylactinomycin. Our data, therefore, stand in agreement with previous findings of Perlman and coworkers who suggested that 4-MHA is derived from 4-methyl-3-hydroxykynurenine (4-MHK) rather than from 3HA. ${ }^{14}$ We have recently cloned the $S$. antibioticus orthologue of the gene acmL encoding 3-hydroxykynurenine (3-HK) methyltransferase from S. chrysomallus (Crnovcic and Keller, unpublished). This gene was designated acmSaL (Acc. no. KF860152). Its translated gene sequence has $85 \%$ identity to AcmL and the sizes of the two proteins are the same. The presence of this gene is a further hint for the possible involvement of 4-MHK in 4-MHA biosynthesis of $S$. antibioticus.

Previous biochemical studies on phenoxazinone formation in actinomycin-producing streptomycetes revealed the presence of phenoxazinone synthase (PHS) in S. antibioticus but not in $S$. chrysomallus $^{\mathbf{3 0}}$ The fact that $S$. antibioticus but not $S$. chrysomallus (or $S$. parvulus) produces hemi-actinomycins and demethyl-hemi-actinomycins suggests that phenoxazinone synthase (PHS) is responsible for synthesis of these novel compounds. PHS has broad substrate specificity by which it is capable to dimerize various structurally different $o$-aminophenols into the corresponding phenoxazinones. ${ }^{1}$ Although PHS was shown to be dispensable for actinomycin synthesis in S. antibioticus,$^{34}$ the data shown here indicate that the presence of this enzyme is correlated to the reaction of abundant 3-HA or 4-MHA with actinomycin halves in contrast to the situation found in S. chrysomallus and S. parvulus. Their phenoxazinone formation is most likely spontaneous ${ }^{3,16}$ and the cellular location of the reaction must be compartmentalized to prevent its overflow with free 4-MHA or 3-HA. ${ }^{15}$ The involvement of PHS in hemi-actinomycin synthesis in $S$. antibioticus would be the first indication of the in vivo involvement of PHS in phenoxazinone formation in $S$. antibioticus and shows that this enzyme can assist in the formation of actinomycins. The real role of PHS is still unknown and it may have a protective role in cellular metabolism by neutralizing excess 4-MHA or other $o$-aminophenols which otherwise are toxic to the cell. ${ }^{35}$ Notwithstanding this, formation of hemi-actinomycins may not be a too severe handicap to $S$. antibioticus since these compounds still exert antimicrobial activity albeit less strongly than actinomycin.

\section{Acknowledgements}

We thank Professor D. Naumann and Dr P. Lasch from the Robert Koch-Institut Berlin, making available for us the Bruker Autoflex instrument to perform the MALDI-TOF measurements. We thank Professor R. Süssmuth from the Teechnische Universität Berlin, making available for us the Exactive ESI-Orbitrap-MS to perform the HPLC-ESI-MS experiments. This work was supported in part by grant Ke452/8-5 and by the DFG Cluster of Excellence 'Unifying Concepts in Catalysis of the Deutsche Forschungsgemeinschaft.

\section{Notes and references}

1 E. Katz, in Antibiotics, ed. P. D. Shaw and D. Gottlieb, Springer, New York, 1967, vol. II, pp. 276-341.

2 U. Keller, J. Biol. Chem., 1984, 259, 8226-8231.

3 U. Keller, J. Biol. Chem., 1987, 262, 5852-5856.

4 W. C. Lima, A. M. Varani and C. F. M. Menck, Mol. Biol. Evol., 2009, 26, 399-406.

5 Y. Hu, V. Phelan, I. Ntai, C. M. Farnet, E. Zazopoulos and B. O. Bachmann, Chem. Biol., 2007, 14, 691-701. Erratum in: Chem. Biol. 2007, 14, 870.

6 W. Li, A. Khullar, S. Chou, A. Sacramo and B. Gerratana, Appl. Environ. Microbiol., 2009, 75, 2869-2878.

7 W. Li, S. Chou, A. Khullar and B. Gerratana, Appl. Environ. Microbiol., 2009, 75, 2958-2963.

8 I. Crnovčić, R. Süssmuth and U. Keller, Biochemistry, 2010, 49, 9698-9705. 
9 U. Keller, M. Lang, I. Crnovčić, F. Pfennig and F. Schauwecker, J. Bacteriol., 2010, 192, 2583-2595.

10 T. W. Giessen, F. I. Kraas and M. A. Marahiel, Biochemistry, 2011, 50, 5680-5692.

11 F. Fawaz and G. H. Jones, J. Biol. Chem., 1988, 263, 4602-4606.

12 E. Katz and H. Weissbach, J. Biol. Chem., 1963, 238, 666-675.

13 H. Weissbach, B. G. Redfield, V. Beaven and E. Katz, Biochem. Biophys. Res. Commun., 1965, 19, 524-530.

14 D. Perlman, S. Otani, K. L. Perlman and J. E. Walker, J Antibiot., 1973, 26, 289-296.

15 I. Crnovčić, J. Vater and U. Keller, J Antibiot., 2013, 66, 211218.

16 U. Keller and W. Schlumbohm, J. Biol. Chem., 1992, 267, 11745-11752.

17 G. H. Jones, J. Biol. Chem., 1993, 268, 6831-6834.

18 F. Arcamone, G. Cassinelli, G. Fantini, A. Grein, P. Orezzi, C. Pol and C. Spalla, Biotechnol. Bioeng., 1969, 11, 1101-1110.

19 U. Keller, H. Kleinkauf and R. Zocher, Biochemistry, 1984, 23, 1479-1484.

20 H. Brockmann and H. Muxfeldt, Chem. Ber., 1958, 91, 12421265.

21 E. Katz, P. Pienta and A. Sivak, Appl. Microbiol., 1958, 6, 236241.

22 E. Bullock and A. W. Johnson, J. Chem. Soc., 1957, 16021607.
23 H. Brockmann, H. Linge and H. Grone, Naturwissenschaften, 1953, 40, 224.

24 K. Ochi, J. Bacteriol., 1982, 150, 598-603.

25 S. Christen, P. T. Southwell-Keely and R. Stocker, Biochemistry, 1992, 31, 8090-8097.

26 H. Suzuki, Y. Furusho, T. Higashi, Y. Ohnishi and S. Horinouchi, J. Biol. Chem., 2006, 281, 824-833.

27 J. C. Freeman, P. G. Nayar, T. P. Begley and J. J. Villafranca, Biochemistry, 1993, 32, 4826-4830.

28 F. Bruyneel, G. Dive and J. Marchand-Brynaert, Org. Biomol. Chem., 2012, 10, 1834-1846.

29 E. Katz and H. Weissbach, J. Biol. Chem., 1962, 237, 882-886.

30 C. E. Barry 3rd, P. G. Nayar and T. P. Begley, Biochemistry, 1989, 28, 6323-6333.

31 H. M. Sobell, S. C. Jain, T. D. Sakore and C. E. Nordman, Nat. New Biol., 1971, 231, 200-205.

32 D. K. Trask and M. T. Muller, Proc. Natl. Acad. Sci. U. S. A., 1988, 85, 1417-1421.

33 A. Bodley, L. F. Liu, M. Israel, R. Seshadri, Y. Koseki, F. C. Giuliani, S. Kirschenbaum, R. Silber and M. Potmesil, Cancer Res., 1989, 49, 5969-5978.

34 G. H. Jones, Antimicrob. Agents Chemother., 2000, 44, 13221327.

35 T. Ishii, H. Iwahashi, R. Sugata and R. Kido, Free Radical Res. Commun., 1991, 14, 187-194. 